\title{
Analyzing calculation results of non-stationary axisymmetric thermoelasticity task for a circular isotropic plate
}

\author{
Dmitriy Shlyahin ${ }^{1, *}$ \\ ${ }^{1}$ Samara State Technical University, Academy of Architecture and Civil Engineering, \\ Molodogvardeyskaya St., 194, Samara, 443001, Russia
}

\begin{abstract}
The paper releases results of numerical calculation of axisymmetric dynamic thermoelasticity task for a fixed circular isotropic plate in case of temperature change on its front faces (boundary conditions of the 1st type). The calculated ratios are obtained by using the GL-theory of thermoelasticity (classical theory), which determines the dependence of the vector of heat flux on the velocity of change and temperature gradient. The mathematical model of the task in question includes differential equations of axisymmetric motion and thermal conductivity, formulated as regard to the component of the movement vector and the function of temperature change. Not self-adjoint system is investigated independently. For its solution, a mathematical apparatus technique of separation of variable in the form of finite integral transformations is used, that is transformations of Fourier, Hankel and generalized integral transformation (GIT). The constructed calculation ratios give an opportunity to define stress and strain state and character of distribution of a thermal field of rigidly fixed circular plate with arbitrary axially symmetrical temperature external influence. It is shown, that elastic inertial characteristics of a plate influence the law of change of movement over time only while investigating very thin plates at high-speed temperature impact.
\end{abstract}

\section{Introduction}

When designing building structures and devices of different purpose, there is a need to study them in conditions of uneven non-stationary heating [1-3]. This effect is accompanied with thermal deformations and stresses, which should be taken into account when analyzing strength characteristics of a certain structure.

Mathematical statement of thermoelasticity tasks includes not self-adjoint linear differential equations of motion and thermal conductivity. Their research is usually carried out separately. Difficulties arising at integrating equations of movement and satisfying boundary conditions, lead to simplification of initial calculation ratios in which inertia forces of elastic system are not taken into account. Besides, the design scheme of such a construction is presented in the form of thin-walled $[4,5]$ or infinitely long bodies $[6,7]$.

\footnotetext{
* Corresponding author: $\underline{\mathrm{d}-612-m i t 2009 @ y a n d e x . r u}$
} 
Another approach is related to the study of the thermal conductivity equation without account of the elastic system deformation $[8,9]$.

\section{Materials and methods}

This research analyzes a rigidly fixed circular isotropic plate, which in a cylindrical system of coordinates occupies the $\left(r_{*}, \theta, z_{*}\right)$ area $\Omega\left\{0 \leq r_{*} b, 0 \leq \theta<2 \pi, 0 \leq z_{*} \leq h^{*}\right\}$, in case of non-stationary axisymmetric of temperature influence on its front faces (see Figure 1): with $z=0 \omega_{1}^{*}\left(r_{*}, t_{*}\right)$, and $z=h^{*} \omega_{2}^{*}\left(r_{*}, t_{*}\right)$

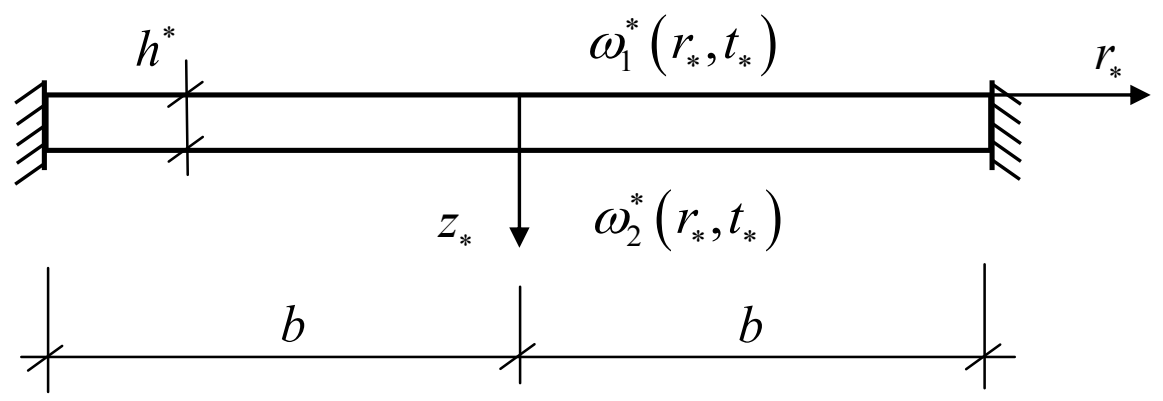

Fig. 1. Design model.

Solutions of this not self-adjoint system of initial differential equations are carried out separately. At the first stage, in the first approximation $(k=1)$, the equation of thermal conductivity concerning the function of temperature change is solved $T^{*}\left(r_{*}, z_{*}, t_{*}\right)$. It is initially assumed that the components of the movement vector $U^{*}\left(r_{*}, z_{*}, t_{*}\right), W^{*}\left(r_{*}, z_{*}, t_{*}\right)$ are equalto zero.

The second step is to solve a task of elasticity with regard to function $U^{*}, W^{*}$ based on a given (well-known) function $T^{*}$ : In the subsequent stages $(k=2,3 \ldots)$ the temperature change function is clarified $T^{*}$. The iteration process ends when the following condition is satisfied:

$$
\left|\left[\int_{0}^{h} T_{k}\left(0, z, t_{\max }\right) d z-\int_{0}^{h} T_{k-1}\left(0, z, t_{\max }\right) d z\right] / T_{k}\left(0, z, t_{\max }\right) d z\right|<\varepsilon \quad\left(\varepsilon=10^{-5}\right)
$$

\section{Equations and mathematics}

The general solution of the problem of thermoelasticity is worked out by the author in Paper [10]. The calculated ratios of the components of movement vector and the temperature change function are as follows: 


$$
\begin{aligned}
& U(r, z, t)=2 \sum_{n=1}^{\infty} \frac{J_{1}\left(j_{n} r\right)}{J_{0}\left(j_{n}\right)^{2}}\left[H_{1}(n, z, t)+\sum_{n=1}^{\infty} G\left(\lambda_{i n}, n, t\right) K_{1}\left(\lambda_{i n}, \mathrm{z}\right)\left\|K_{i n}\right\|^{2}\right] \\
& W(r, z, t)=W_{1}(t)+2 \sum_{n=0}^{\infty} \frac{J_{0}\left(j_{n} r\right)}{J_{0}\left(j_{n}\right)^{2}}\left[H_{2}(n, z, t)+\sum_{n=1}^{\infty} G\left(\lambda_{i n}, n, t\right) K_{2}\left(\lambda_{i n}, \mathrm{z}\right)\left\|K_{i n}\right\|^{2}\right] \\
& T(r, z, t)=2 \sum_{j=0}^{\infty} \frac{J_{0}\left(j_{n} r\right)}{J_{0}\left(j_{n}\right)^{2}}\left[f_{1}(z) p_{1 H}+f_{2}(z) p_{2 H}+\frac{2}{h} \sum_{m=1}^{\infty} L_{H}(n, \mathrm{~m}, t) \sin \left(\lambda_{m}, \mathrm{z}\right)\right]
\end{aligned}
$$

where we see standardization functions, load transformants, components of the core of final integral transformation method; the normalizing constant of the generalized integral transformation.

\section{Conclusions}

As an example, a rigidly fixed circular reinforced concrete plate $\left(b=1 \mathrm{~m}, T_{0}=20^{\circ} \mathrm{C}\right.$, $E=2 \times 10^{10} E=2 \times 10^{10} \mathrm{~Pa}, v=0.2, \rho=2000 \mathrm{~kg} / \mathrm{m}^{3}, L=1.75 \mathrm{w} /\left(\mathrm{m}^{\circ} \mathrm{C}\right), \alpha_{t}=1.2$ $\left.\times 10^{-5} 1 /{ }^{\circ} \mathrm{C}, \mathrm{k}=0.76 \times 10^{-6} \mathrm{~m}^{2} / \mathrm{s}\right)$ in case of action on the upper front face $\left(z_{*}=0\right)$ of the temperature load in the form of:

$$
\omega_{I}^{*}\left(r_{*}, t_{*}\right)=\left(1-r_{*}\right) T_{\max }\left[\sin \left(\frac{\pi}{2 t_{\max }} t_{*}\right) H\left(t_{\max }-t_{*}\right)+H\left(t_{\max }-t *\right)\right], \omega_{2}^{*}\left(r_{*}, t_{*}\right)=0,
$$

where $H(\tilde{t})$-is the Heaviside step function $(H(\tilde{t})=1$ at $(\tilde{t}) \geq 0, H(\tilde{t})=0$ at $(\tilde{t})<0)$, $T_{\text {max }}=T_{\text {max }}^{*}-T_{0}, T_{\text {max }}^{*}, t_{\max }-$ is the maximum value of external temperature effect and its corresponding time $\left(T_{\max }^{*}=100^{\circ} \mathrm{C}\right)$.

The structures of different thickness were investigated at high-speed temperature loading $t_{\text {max }}=0.03 c$.

Figures $2-4$ demonstrate numerical results of the calculation, on the basis of which the following conclusions can be drawn:

1. At high-speed temperature loading, inertial characteristics of the elastic system in thin plates $\left(h^{*}=0.01(m)-\right.$ Figure 2 , solid line $)$ have an effect on the graph the axial component of the movement vector change $W^{*}$ over time $t_{*}$. In thicker structures $\left(h^{*}=\right.$ $0.1(m)-$ Figure 2 , dashed line) of this dependence is not observed (the character of change over time $W^{*}$ and $\omega_{1}^{*}$ coincide), and in this case quasi-static equations of equilibrium (time $t_{*}$ is a parameter) can be used while studying the problem of elasticity theory.

2. The highest direct stresses $\sigma_{r r}$ are observed on the plate front face $z_{*}=0$ (see Figure 3, Graph 1). This component of stress decreases together with thickness (see Fig. 3, Graph 2) and is virtually absent $z_{*}=h^{*}$. The size and nature of the distribution of normal stresses are not substantially dependent on the thickness of the plate $h^{*}$.

3. In construction, with thickness $h^{*}=0.1(\mathrm{~m})$, its temperature field along its height is distributed according to a linear dependence (see Figure 4, Graph 1), and if the height of the plate $h^{*}=0.3(\mathrm{~m})$ increases, its temperature field is distributed according to a parabolic dependence (see Figure 4, Graph 2). The deformation of the plate does not affect the ${ }^{\prime \prime} T^{*}\left(0, z_{*}, t_{\text {max }}\right)-z_{*}{ }^{\prime \prime}$ dependence. 


$$
W^{*}\left(0,0, t_{*}\right), \mathrm{m}
$$

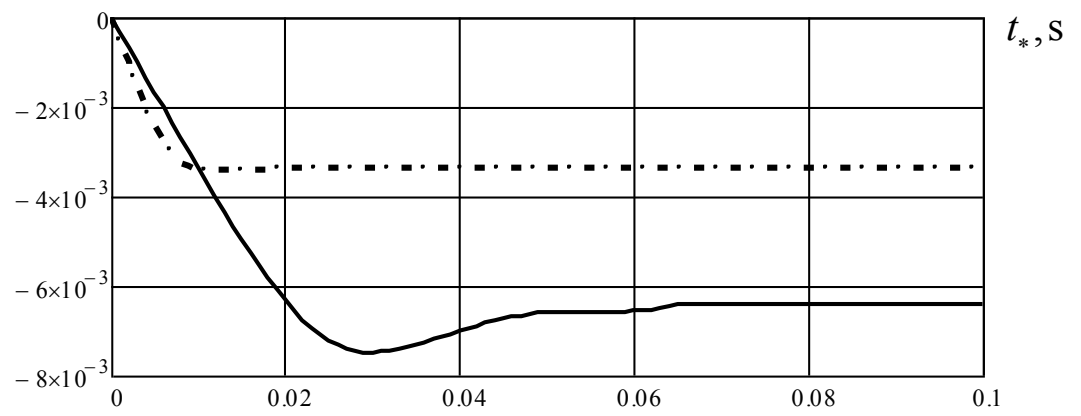

Fig. 2. Time change $t_{*}$ graphs $W\left(0,0, t_{*}\right)\left(h^{*}=0.01(m)-\right.$ solid line, $h^{*}=0.1(m)-$ dashed line (numerical results are 5 times increased))

$$
\sigma_{r r}\left(r_{*}, z_{*}, t_{\max }\right) \times 10^{-7}, \mathrm{H} / \mathrm{m}^{2}
$$

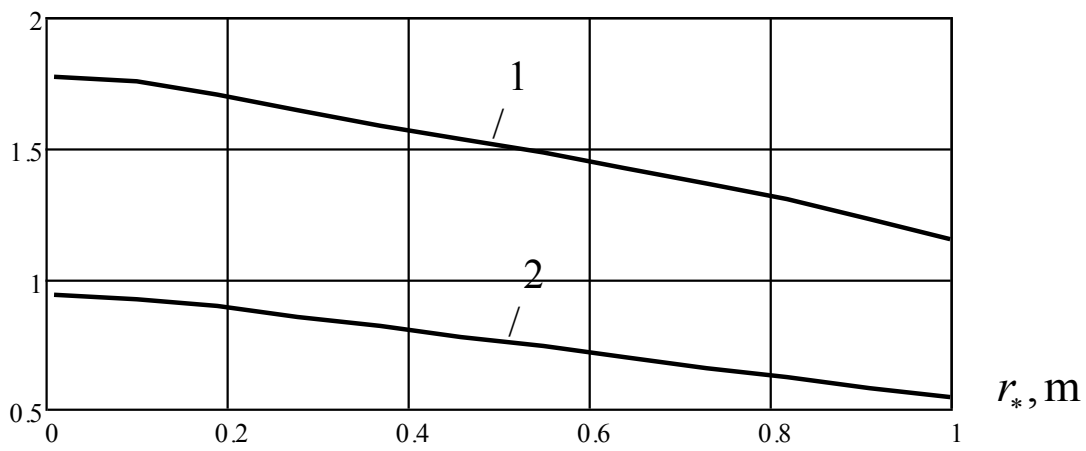

Fig. 3. Radial coordinate $r_{*}$ change $\sigma_{r r}\left(r_{*}, z_{*}, t_{\max }\right)\left(h^{*}=0.01 \mathrm{~m}, 1-\mathrm{z}_{*}=0,2-\mathrm{z}_{*}=\mathrm{h}^{*} / 2\right)$

$$
T^{*}\left(0, z_{*}, t_{\max }\right),{ }^{0} C
$$

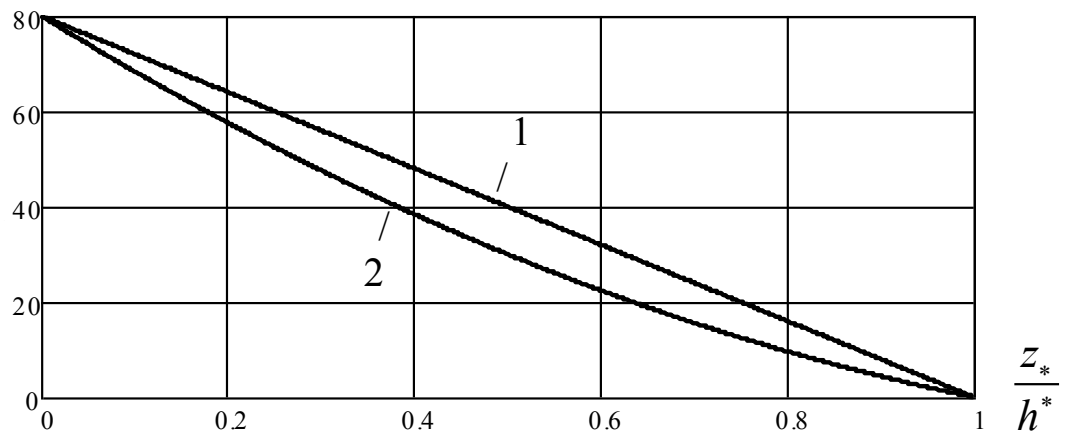

Fig. 4. Change $T^{*}\left(0, z_{*}, t_{\max }\right)$ according to the height of the plate $\left(1-h^{*}=0.1 m, 2-h^{*}=0.3 \mathrm{~m}\right)$ 


\section{References}

1. P.V. Tsoi, System methods for calculating heat and mass transfer tasks (Moscow, 2005)

2. A.G. Shashkov, V.A. Bubnov, S.Yu. Yanovskiy, Wave effects of thermal conductivity: system and structural approach (Moscow, 2004)

3. SP 27.13330.2011. Concrete and reinforced concrete structures designed for operation in high temperature conditions, Moscow, 116 (2011)

4. Yu.N. Radaev, D.A. Semenov, Vestnik of Samara State University, Natural-science series, No. 8/2 (67), 109-129 (2008)

5. V. A. Kovalev, Yu. N. Radaev, R.A. Revinskiy,News of Saratov Univ. Mathematics. Mechanics. Informatics, 11 (2011)

6. V.A. Kudinov, Veslnik SamGTU. Physics-Math Science, Issue 2 (35), 130-135 (2014)

7. P.V. Zhukov, Vestnik ISEU, 3, 1-4 (2013)

8. V.A. Kudinov, E.M. Kartashev, V.V. Kalashnikov, Analytical solutions of problems of heat and mass transfer and thermoelasticity for multilayer constructions, Moscow, High School, 430 (2005)

9. V.A. Kudinov, R.M. Klebneev, E.A. Kuklova, Vestnik SamGTU. Physical-Math Science, 21, No. 1, 197-206 (2017)

10. D.A. Shlyakhin, MATEC Web of Conferences, 117 (2017) 